\title{
Trends and practices in prescribing anti-psychotropic medications in hospitalized patients with psychiatric disorders in a secondary care hospital
}

\author{
Javedh Shareef*, Padma G. M. Rao, Itaf Mohamad Ayman
}

Department of Clinical Pharmacy and Pharmacology, RAK College of Pharmaceutical Science, Ras Al Khaimah, United Arab Emirates

Received: 29 August 2020

Accepted: 05 October 2020

*Correspondence:

Dr. Javedh Shareef,

Email: javedh@rakmhsu.ac.ae

Copyright: (c) the author(s), publisher and licensee Medip Academy. This is an open-access article distributed under the terms of the Creative Commons Attribution Non-Commercial License, which permits unrestricted non-commercial use, distribution, and reproduction in any medium, provided the original work is properly cited.

\begin{abstract}
Background: Advances in the psycho-pharmacotherapy enhance the development of newer and better drugs in the management of psychiatric disorders. However, their proper utilization, safety and efficacy and adverse effects in the clinical practice needs continuous study. The study aimed to assess the trends and practice of prescribing psychotropic medications in hospitalized patients in a secondary care hospital in Ras Al Khaimah.

Methods: A prospective observational study was carried out for a period of six months in a psychiatry department. All the patient details including the demographic data and prescribing pattern of antipsychotic medication were collected from the patient case records and were later analysed by using descriptive statistics.

Results: A total of 50 patient's prescription were analysed during the study period. Male (54\%) predominance was noted over females $(46 \%)$ with majority $(64 \%)$ of patients were in the age group of 21-40 years. Schizophrenia $(35.8 \%)$ was the most common psychiatric disorders followed by affective disorders $(30.86 \%)$. The average number of psychiatric drugs per prescription was found to be $3.38 \pm 1.23$. Antipsychotics (43.36\%) were the commonly prescribed class of medications followed by mood stabilizers $(12.38 \%)$ and anxiolytics $(11.06 \%)$ with olanzapine $(n=26)$, sodium valproate $(n=21)$ and clonazepam $(n=9)$ being frequently prescribed medications. Escitalopram $(n=9)$ was the most commonly used anti-depressants. Combination therapy (86\%) is preferred over monotherapy (14\%).

Conclusions: This study helps to assist in ensuring rational drug therapy and reducing the incidence of drug related problems and medication errors and thereby enhancing the quality of care in patients with psychiatric disorders.
\end{abstract}

Keywords: Prescribing pattern, Psychotropic medications, Inpatients, Psychiatric disorders

\section{INTRODUCTION}

Psychiatric disorders are one of the most common causes of morbidity and form an important public health priority. It affects the individual's mental health leading to changes in emotion, thinking or behavior causing a high burden of disorders such as anxiety, depression, bipolar disorders, schizophrenia and other psychiatric illness. ${ }^{1}$ Patients diagnosed with chronic disorders such as cancer, diabetes, cardiovascular disease, stroke are often affected with mental disorders and there is evidence that even mental disorders predispose the patients to myocardial infarction and diabetes, both of which conversely increase the likelihood of psychiatric disorders. Mental health illness contributes to poor health outcomes, die prematurely and often experience severe human rights violations, discrimination and stigma. Untreated or long standing chronic physical illness, low socioeconomic status, alcohol use and stress are some of the common risk factors to develop mental disorders. ${ }^{2,3}$ 
It has been estimated that 450 million people worldwide are suffering at any given time from some kind of mental or brain disorder, including behavioural and substance abuse disorders. It is also well known that four of the ten leading causes of disability worldwide are neuropsychiatric disorders, accounting for $30.8 \%$ of total disability and $12.3 \%$ of the total burden of disease and is expected to rise to $15 \%$ by the year $2020 .{ }^{4}$

Remarkable advances in the psychosocial and pharmacotherapy with evidence-based interventions provides an opportunity for people with mental disorders to live a normal and productive life. However, prescribing psychotropic medications is complex and often challenging to clinicians with the availability of enormous wide variety of drugs with complicated regimen and varying dosage forms. ${ }^{5}$ Individuals diagnosed with mental disorders often have medical comorbidities exposing them to receive multiple medications with narrow therapeutic index leading to polypharmacy. The most common reason behind the use of polypharmacy is to boost or augment the efficacy of the primary drug, to prevent or manage the adverse effects of primary drug or to treat the comorbid illness. ${ }^{6,7}$

Drug utilization studies continues to monitor and evaluate the prescribing patterns to ensure the care provided are evidence based, rational and cost-effective with least number of medications. ${ }^{8}$ The selection of the drugs from the different class of medication will be based on the therapeutic effectiveness, adverse drug reactions, sociodemographic characteristics of the patients and the prescription setting (hospital, community, private or public). Although psychotropic drugs have an extraordinary impact on psychiatry, their utilization, effectiveness and safety in the real life actual clinical practice situation needs continuous study. ${ }^{1,9}$ Hence the study was carried out with the aim to assess the trends and practice of prescribing psychotropic medications in hospitalized patients in a secondary care hospital in Ras Al Khaimah.

\section{METHODS}

It was a prospective, cross-sectional observational study carried out for a period of six months in hospitalized patients diagnosed with psychiatric disorders admitted under the psychiatry department of a secondary hospital in Ras Al Khaimah. The ethics and research committee of the medical and health science university of Ras $\mathrm{Al}$ Khaimah and the ministry of health and prevention approved this study. All the patients of either gender diagnosed with psychiatric disorder and receiving one or more antipsychotic drug were included in the study. Patients visiting the outpatient psychiatry department, patients treated with non-pharmacological therapy, history of drug abuse, patients who are agitated and uncooperative and patients with incomplete medical records were excluded from the study. All the patients admitted in the psychiatry department were followed and all the necessary details such as demographic and clinical data including diagnosis and co-morbidities along with the drug related information were collected and documented in a pre-structured, customized data collection form designed as per the need of the study. The collected data were later entered into Microsoft excel and SPSS version 24 for windows to analyze the data for the trends and practice of prescribing psychotropic medications in the hospital. Descriptive statistics for continuous variables were expressed as mean and standard deviation (Mean \pm SD). Categorical variables were described as frequencies with percentages for the total study population. All the findings were summarized by using tables and figures.

\section{RESULTS}

This section represents a comprehensive summary of the data collected by the researcher in the tabular and graphical form using descriptive statistics. A total of 50 prescriptions of hospitalized patients with psychiatric disorders were studied for various parameters during the study period. The percentage of male and female patients was $54 \%(n=27)$ and $46 \%(n=23)$ respectively. The mean age of the study sample was $37.48 \pm 12.24$, with majority of the patients in the age group 21-40 years [32 (64\%)] followed by 41-60 years [15 (30\%)] and [1 (2\%)] of the study population were $\leq 20$ years of age.

Morbidity pattern among the psychiatric illness identified during the study period showed schizophrenia was the most common disorder encountered $(35.80 \%)$ followed by affective disorders $(30.86 \%)$ including both mania and depression. Anxiety disorders included $13.58 \%$ of the study population and the other psychiatric disorders $(19.75 \%)$ were acute psychosis $(16.04 \%)$, personality disorder $(1.23 \%)$, abnormal behaviour (1.23\%) and autism spectrum disorder $(1.23 \%)$. In the present study, more than half $(52 \%)$ of the study populations was having no co-morbidities, $26 \%$ were having one comorbidity, $18 \%$ were having two co-morbidities and $4 \%$ were having three or more co-morbidities. The most common co-morbidities identified among the study population were diabetes mellitus including gestational diabetes, cardiovascular diseases, stroke, respiratory diseases, thyroid disorder, benign prostrate hypertrophy, infectious disease and carcinoma.

The demographic details of the study populations with psychiatric disorders is shown in the following (Table 1).

A total of 226 drugs including both psychiatric and nonpsychiatric drugs were prescribed during the study period. The mean number of drugs per prescription was found to be $4.52 \pm 1.36$ (Mean \pm SD). Among the total number of drugs received by the study populations, majority $(68 \%)$ of the patients were receiving 4-6 drugs per prescription followed by $30 \%$ of the patients receiving 1-3 drugs and $2 \%$ of the patients were prescribed with seven or more drugs per prescription. 
Among the total drugs, $74.77 \%(n=169)$ were psychiatric medications and the mean number of psychiatric medications per prescription was found to be $3.38 \pm 1.23$ $($ Mean \pm SD). Considering the route of administration of the drugs prescribed, most of the drugs prescribed were oral medications $(86.72 \%)$ followed by $11.94 \%$ of the medications were injectable drugs. Topical and inhalational were the other route of administration for the prescribed medications. Drug use pattern in psychiatric study populations is shown in the following (Table 2).

Table 1: Demographic details of the study populations.

\begin{tabular}{|ll|}
\hline Characteristics & Total $(\mathbf{n = 5 0})(\%)$ \\
\hline Gender & $27(54)$ \\
\hline Fale & $23(46)$ \\
\hline Age (years) & $1(2)$ \\
\hline$\leq 20$ & $32(64)$ \\
\hline $21-40$ & $15(30)$ \\
\hline $41-60$ & $2(4)$ \\
\hline $61-80$ & \\
\hline Types of psychiatric illness & $11(13.58)$ \\
\hline Anxiety disorders & $25(30.86)$ \\
\hline Affective disorders & $29(35.80)$ \\
\hline Schizophrenia & $16(19.75)$ \\
\hline Other psychiatric illness & \\
\hline Co-morbidities & $26(52)$ \\
\hline Nil & $13(26)$ \\
\hline 1 & $9(18)$ \\
\hline 2 & $2(4)$ \\
\hline$\geq 3$ & $15(30)$ \\
\hline No. of drugs received by patient per prescription \\
\hline $1-3$ & $34(68)$ \\
\hline $4-6$ & $1(2)$ \\
\hline$\geq 7$ & \\
\hline
\end{tabular}

Table 2: Drug usage pattern in psychiatric illness.

\begin{tabular}{|ll|}
\hline Drug use indicators & Results \\
\hline $\begin{array}{l}\text { Total number of prescriptions } \\
\text { analysed }\end{array}$ & 50 \\
\hline Total number of drugs prescribed & 226 \\
\hline $\begin{array}{l}\text { Average number of drugs per } \\
\text { prescription (Mean } \pm \text { SD) }\end{array}$ & $4.52 \pm 1.36$ \\
\hline $\begin{array}{l}\text { Total number of psychiatric drugs } \\
\text { prescribed }\end{array}$ & 169 \\
\hline $\begin{array}{l}\text { Average number of psychiatric } \\
\text { drugs per prescription (Mean } \pm \text { SD) }\end{array}$ & $3.38 \pm 1.23$ \\
\hline $\begin{array}{l}\text { Percentage of injectable drugs } \\
\text { prescribed (\%) }\end{array}$ & $27 / 226(11.94)$ \\
\hline
\end{tabular}

Regarding the prescribing pattern of anti-psychotic medications, combination therapy $(86 \%)$ was more preferred than monotherapy (14\%) among the study populations. The prescribing pattern showing the practice of monotherapy and combination therapy is graphically represented in the following (Figure 1).

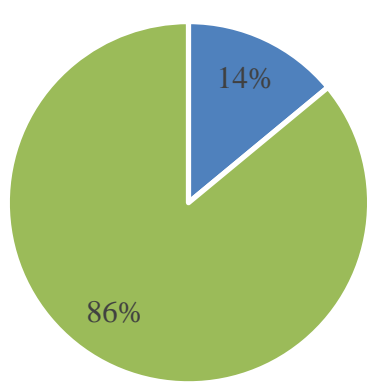

- Monotherapy - Combination therapy

Figure 1: Prescribing pattern of the practice of monotherapy and combination therapy.

All the psychiatric drugs prescribed were categorized into 4 broad classes namely antidepressants, antipsychotics, anxiolytics and anti-mania/mood stabilizers. The category 'others' includes non-psychiatric medications that were prescribed to the study populations. Among the psychiatric medications prescribed, antipsychotics $(43.36 \%)$ were the most commonly prescribed class of medications followed by anti-mania/mood stabilizers (12.38\%), anxiolytics (11.06\%) and antidepressants (10.61\%). Anti-cholinergic account for $2.21 \%$ of the total medications prescribed among the study populations. In the other category of medications, proton pump inhibitors $(3.09 \%)$ followed by metformin $(2.21 \%)$ were the most commonly prescribed medications. The graphic representation of the prescription wise distribution of psychiatric and non-psychiatric medications received by the study populations is shown in following (Figure 2).

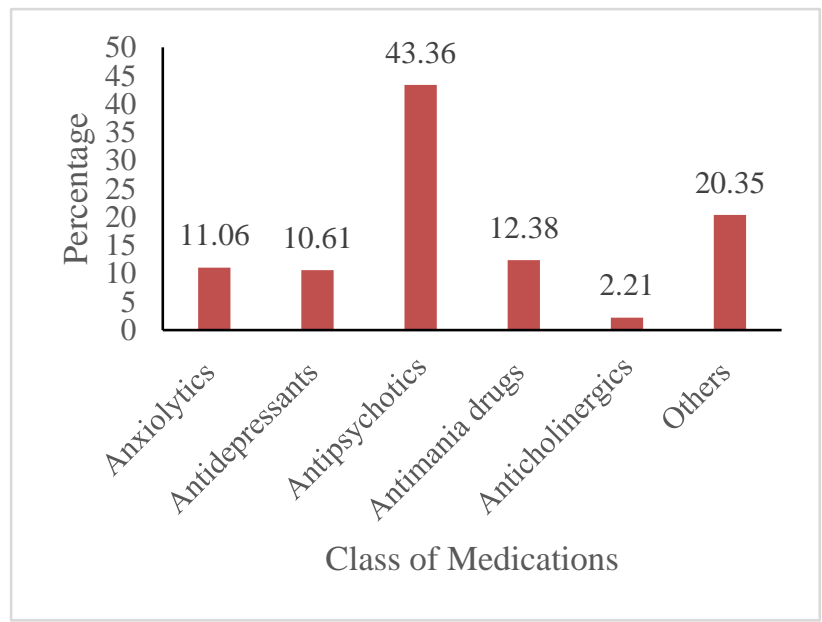

Figure 2: Prescription wise distribution of psychiatric and non-psychiatric medications received by the study populations.

The prescribing frequency of various class of psychiatric medications were analysed. Among the antipsychotic medications, olanzapine $(n=26)$ and haloperidol $(n=15)$ 
belonging to the class of typical and atypical antipsychotics were the most commonly prescribed medications. Escitalopram $(n=9)$ belonging to the class of selective serotonin reuptake inhibitor (SSRI) were the most commonly prescribed antidepressants. Amitriptyline $(n=2)$ is the only tricyclic antidepressant that has been used in the study populations. Among the benzodiazepines and mood stabilizers, clonazepam $(n=9)$ and sodium valproate $(n=21)$ were the most commonly prescribed medications among the study populations respectively. The prescribing prevalence of the individual psychiatric medications is shown in following (Table 3 ).

Table 3: Prescribing prevalence of individual psychiatric medications.

\begin{tabular}{|c|c|c|c|}
\hline S. no. & Drug class & Name of the medications & Frequency and percentage $(n=169)$ \\
\hline \multirow{4}{*}{1.} & \multirow{4}{*}{ Benzodiazepines } & Clonazepam $(\mathrm{n}=9)$ & \multirow{4}{*}{$18(10.65)$} \\
\hline & & Diazepam $(n=5)$ & \\
\hline & & Lorazepam $(\mathrm{n}=2)$ & \\
\hline & & Bromazepam $(n=2)$ & \\
\hline 2. & Non-benzodiazepines & Zaleplon $(n=1)$ & $01(0.59)$ \\
\hline \multirow{3}{*}{3.} & \multirow{3}{*}{ Typical antipsychotics } & Chlorpromazine $(n=9)$ & \multirow{3}{*}{$26(15.38)$} \\
\hline & & Haloperidol $(n=15)$ & \\
\hline & & Trifluoperazine $(n=2)$ & \\
\hline \multirow{8}{*}{4.} & \multirow{8}{*}{ Atypical antipsychotics } & Risperidone $(\mathrm{n}=15)$ & \multirow{8}{*}{$72(42.60)$} \\
\hline & & Quetiapine $(\mathrm{n}=13)$ & \\
\hline & & Olanzapine $(n=26)$ & \\
\hline & & Aripiprazole $(n=12)$ & \\
\hline & & Sertindole $(\mathrm{n}=2)$ & \\
\hline & & Paliperidone $(n=2)$ & \\
\hline & & Ziprasidone $(\mathrm{n}=1)$ & \\
\hline & & Clozapine $(\mathrm{n}=1)$ & \\
\hline 5. & Tricyclic antidepressants & Amitriptyline $(n=2)$ & $02(1.18)$ \\
\hline \multirow{6}{*}{6.} & \multirow{6}{*}{$\begin{array}{l}\text { Selective serotonin reuptake } \\
\text { inhibitor (SSRI) }\end{array}$} & Escitalopram $(n=9)$ & \multirow{6}{*}{$20(11.83)$} \\
\hline & & Fluoxetine $(n=5)$ & \\
\hline & & Sertraline $(n=3)$ & \\
\hline & & Mirtazapine $(\mathrm{n}=1)$ & \\
\hline & & Fluvoxamine $(n=1)$ & \\
\hline & & Vortioxetine $(\mathrm{n}=1)$ & \\
\hline 7. & $\begin{array}{l}\text { Selective-norepinephrine } \\
\text { reuptake inhibitors }\end{array}$ & Venlafaxine $(n=2)$ & $02(1.18)$ \\
\hline \multirow{4}{*}{8.} & \multirow{4}{*}{ Mood stabilizers } & Lithium $(n=4)$ & \multirow{4}{*}{$28(16.56)$} \\
\hline & & Sodium valproate $(n=21)$ & \\
\hline & & Carbamazepine $(\mathrm{n}=2)$ & \\
\hline & & Topiramate $(\mathrm{n}=1)$ & \\
\hline
\end{tabular}

Table 4: Usage of injectable drugs in study populations.

\begin{tabular}{|llll|}
\hline S. no. & Drug class & Name of the medications & Frequency and percentage $(\mathbf{n = 2 7})$ \\
\hline 1. & Benzodiazepines & Diazepam & 5(18.51) \\
\hline 2. & Typical antipsychotics & Haloperidol & $11(40.74)$ \\
\cline { 3 - 4 } & Chlorpromazine & $6(22.22)$ \\
\hline \multirow{2}{*}{ 3. } & Atypical antipsychotics & Risperidone & $2(7.40)$ \\
\hline 4. & Insulin preparations & Paliperidone & $1(3.70)$ \\
\hline 5. & Anti-histamines & Insulin regular & $1(3.70)$ \\
\hline
\end{tabular}

Among the various injectable drugs used in the study populations, haloperidol $[n=11(40.74 \%)]$ is the most commonly used antipsychotic medications followed by chlorpromazine $[n=6(22.22 \%)]$ and diazepam $[n=5$ $(18.51 \%)]$. Insulin and promethazine were the nonpsychiatric parenteral preparations used among the study populations. The frequency and percentage of the various parenteral medications used among the study population is shown in the following (Table 4).

\section{DISCUSSION}

In the present study, male preponderance was noted over females. However, this similarity was not seen by many 
researchers which showed an increase in number of female patients visiting the psychiatry department as compared to men. ${ }^{10-12}$ The mean age of the study population was found to be $37.82 \pm 11.83$ and the age group between 21-40 years accounted for the majority of all the psychiatric disorders and has been seen in other studies. ${ }^{12,13}$ The increased prevalence of psychiatric disorders in this age group is due to a transition from childhood and adolescence to adulthood, increasing the susceptibility for higher level of stress because of the demands of their social role. A study carried in India showed that the prevalence of schizophrenia is common among the adolescence aged between 20-40 years of age. ${ }^{14}$ Patients aged 60 and above comprised $4 \%$ of the study population. It is observed that in patients aged 60 and above psychiatric disorders goes undetected and sometimes get confused with natural ageing process.

Morbidity pattern of psychiatric illness among the study population showed that schizophrenia $(35.80 \%)$ is the most common psychiatric disorder followed by affective disorders (30.86\%) and other psychiatric disorders $(19.75 \%)$. Similar observations were also seen in studies carried out by other researchers which showed that schizophrenia and delusional disorder was the major diagnosis among the study population followed by bipolar disorder. ${ }^{15-17}$ More than half $(52 \%)$ the number of study populations did not have any co-morbidities and $26 \%$ of them had one co-morbidity, $18 \%$ with two comorbidities and $4 \%$ had three or more co-morbidities. The most common co-morbidities identified in the study population were diabetes mellitus including gestational diabetes mellitus followed by hypertension and dyslipidaemia. In few cases, patients diagnosed with diabetes mellitus were also receiving antipsychotics such as risperidone and clozapine. It is well from the published literature that prolonged use of atypical antipsychotics is associated with three times higher incidence rate of metabolic syndrome along with the increase in the weight and body mass index. ${ }^{18}$ Whether they developed diabetes after the initiation of anti-psychotics or before and had any family history of diabetes mellitus is not clear from the case records. The other co-morbidities involve two cases of benign prostrate hypertrophy and one case each of asthma, stroke, epilepsy, thyroid disorders, cancer, renal disease and acute bronchitis. These finding resemble with an Indian study which showed that about one-third $(30.4 \%)$ patients had one or more physical comorbidities involving neurological, cardiovascular and endocrinological disorders. ${ }^{19}$ Analysis of the prescription pattern showed that the average number of prescription was $3.38 \pm 1.23$ which is almost similar to other studies where it ranged from 1.07 to 3.39 drug per prescription. ${ }^{10,12,13,16,17}$ Among the 50 patients monitored in our study, majority $(68 \%)$ of the patients were receiving at an average of 4-6 drugs per prescription followed by $30 \%$ with 1-3 medications and $2 \%$ of the patients were receiving seven or more drugs per prescription. Average number of drugs per prescription is an important part of prescription audit. It is preferable to keep the number of prescriptions to the lowest minimum possible, but in our study two-third of the patients are receiving three or more medications suggesting a trend of polypharmacy. This may be attributed to the complexity of presentation in psychiatric illness and the presence of multiple co-morbidities by study populations. However, polypharmacy can lead to medication non-adherence, drug interactions, adverse drug reactions, improper use of effective treatments, healthcare expenditure and medication errors. Considering the prescribing pattern of antipsychotic medications in our study, it was identified that majority $(86 \%)$ of the patients were prescribed with combination therapy as compared to monotherapy (14\%) which is comparable to the findings of other studies. ${ }^{14,17,20}$ Even though the guidelines recommend initiating the treatment with only one antipsychotic medication at a time, many of the times the use of combination of antipsychotic medications might be required to achieve greater therapeutic responses when the use of single antipsychotic drug provides unsatisfactory response and also due to the complexity of the clinical presentation.

Prescription wise distribution of class of medications received by the study population showed that antipsychotics $(43.36 \%)$ is the most commonly prescribed followed by anti-mania /mood stabilizers (12.38\%), anxiolytics $(11.06 \%)$, antidepressants $(10.61 \%)$ and anticholinergics $(2.21 \%)$. This could be due to the diagnostic profile of patients, as most of the patients in our study was diagnosed to have psychotic disorders. Similar finding was also observed in other studies carried out on prescribing pattern of antipsychotic medications. ${ }^{11,15,19,21}$ But the various studies carried out in an outpatient department of psychiatric hospital showed that anxiolytics followed by sedative-hypnotics, antidepressants and antipsychotics were the most commonly prescribed medications. ${ }^{13,22-24}$ The 'others' category $(20.35 \%)$ includes oral non-psychiatric medications such as oral hypoglycaemic agents, antihypertensive, acid suppressive agents, dyslipidaemia agents, antiplatelet agents, infectious agents, vitamins and nutrition supplements and cough preparations which were co-prescribed with the psychiatric medications associated with their co-morbid conditions. Topical preparations were prescribed in two cases and inhalation medications was provided in one case.

Among the antipsychotic medications, significant proportion of patients were on atypical antipsychotics $(42.6 \%)$ as compared to typical antipsychotics $(15.38 \%)$. This preponderance of atypical antipsychotics was also seen in many studies and could be due to claims of better safety and tolerability profile, treatment of negative symptoms as well as drug resistant cases of schizophrenia and lower incidence of extra pyramidal adverse effects. ${ }^{15,19,24}$ But prolonged use of atypical antipsychotics can cause weight gain, glucose intolerance, dyslipidaemia, myocarditis and cardiomyopathy. As similar to the trend seen in other 
studies, olanzapine $(\mathrm{n}=26)$ followed by haloperidol $(n=15)$ and risperidone $(n=15)$ were the most commonly prescribed antipsychotics among the study populations..$^{10,13,25,26}$ In the present study, only one prescription of clozapine $(n=1)$ has reported which was similar to the previous studies that reported a lower rate of prescription of clozapine..$^{13,15,27}$ Majority of the patients with bipolar disorder were prescribed with mood stabilizers and sodium valproate $(n=21)$ is the most commonly prescribed anti-mania medication as compared to lithium $(n=4)$ in the present study. This may be due to the patient's characteristics, physician preference, adverse effects profile of the medication and ease in initiating valproate. Though the guidelines recommend lithium as the main stay of the treatment as compared to anticonvulsant, concerns about the low therapeutic index and periodic determination of serum drug concentration explains the lesser utilization of lithium as compared to other agents. Similar observation has been reported in many prescribing pattern studies of antipsychotic medications. ${ }^{13,19,22}$ In few cases, mood stabilizers with antiepileptic properties such as carbamazepine $(n=2)$ and topiramate $(n=1)$ were also prescribed to manage their comorbid epilepsy. In the present study, benzodiazepines are the most common class of drugs prescribed for the treatment of anxiety disorders because of its rapid onset of action, immediate relief of the symptoms and good efficacy. It is also used as adjuncts to anti-depressants and anti-psychotics to improve insomnia, agitation and psychotic symptoms. Clonazepam $(n=9)$ being the most common prescribed one followed by diazepam $(n=5)$ possibly because of its potential to act as a psychotherapeutic adjunct in different psychiatric conditions. It is always recommended to use a short acting benzodiazepine as continuous and prolonged use of longer acting benzodiazepines can result in memory impairment, tolerance, dependence and may develop withdrawal symptoms when the dose is decreased or discontinued. Guidelines recommend the use of benzodiazepines for short term or intermittent courses in minimum effective doses depending on the severity of the symptoms. These observations were similar to the findings of other drug utilization pattern studies carried out in various settings. ${ }^{13,19,22,24}$ Zaleplon, a nonbenzodiazepine derivative was prescribed in one case and the reason for switching to a non-benzodiazepine could not be identified from the case records.

With regards to anti-depressants, selective serotonin reuptake inhibitors (SSRIs) such as escitalopram $(n=9)$, fluoxetine $(n=5)$ and sertraline $(n=3)$ are the most commonly prescribed medications in our study as compared to tricyclic antidepressants. This finding is similar to the trend those reported by other studies. ${ }^{10,22-24}$ SSRI are generally considered as safe at higher doses with lesser adverse effects and better tolerability accounts for their popularity being the most widely prescribed antidepressants. Also, the current guidelines recommend the use of SSRI as the first line agent in patients with depression. Anti-cholinergic such as trihexyphenidyl accounts for $2.21 \%$ of the study populations. It is usually co-prescribed with antipsychotics for various psychiatric illness due to their protective effect against extrapyramidal side effects (EPS) of the neuroleptic drugs. Our study has shown a lower prescription rate of anticholinergics as compared to other studies that showed a range between 5.5-94\%.14,20,22,24,27,28 World health organization treatment guidelines do not recommend routine use of anticholinergics in patients with psychiatric illness who are on antipsychotics but use only for short term in selected cases. it should only be used in patients who are on high potency antipsychotics, having a previous history of extrapyramidal side effects or develops EPS and showing no improvement even after switching to another antipsychotic drug. Considering the total 226 medications prescribed in the study populations, $11.94 \%(n=27)$ medications were prescribed as parenteral preparations. This finding is higher than that found in similar studies where it ranged from $0.03-6.2 \% .^{11,13,17,25}$ Haloperidol $(40.74 \%)$ is the most commonly used parenteral depot preparations in our study. Our findings are in line with the studies examining the use of psychotropic medications in schizophrenia patients in other countries. ${ }^{15,23,24}$ Depot parenteral preparations of antipsychotics are useful in the management of acute phases of schizophrenia for the rapid control of the symptoms and are also found to be useful in cases of patient non-compliance with the treatment and failure with oral medications.

\section{CONCLUSION}

The study provides an insight on present trends in prescribing patterns of psychiatric medications in hospital settings. It will also assist in ensuring rational drug therapy and reducing the incidence of drug related problems and medication errors and thereby enhancing the quality of care in patients with psychiatric disorders. Further studies involving larger sample size for a longer duration may help to provide information for improving psychotropic drug utilization in mental health care facilities.

\section{ACKNOWLEDGEMENTS}

Authors would like to thank to the president and ethics and research committee of the medical and health science university of Ras Al Khaimah and the ministry of health and prevention for the approval and giving permission to conduct the study. Also thankful to the head, department of psychiatry of Ibrahim Bin Hamad Obaidullah hospital and the dean, Ras Al Khaimah college of pharmaceutical sciences for providing necessary support and encouragement to carry out the study.

\author{
Funding: No funding sources \\ Conflict of interest: None declared \\ Ethical approval: The study was approved by the \\ Institutional Ethics Committee
}




\section{REFERENCES}

1. Swamy MK, Wagle L, Giri V. Drug utilization pattern of psychotropic drugs in psychiatric outpatient department of rural tertiary care teaching hospital. Int J Pharmaceut Sci Res. 2016;7(8):34973508.

2. Overview of the global situation. Mental Health Action Plan 2013- 2020. Available at: https://apps.who.int/iris/bitstream/handle/10665/899 66/9789241506021_eng.pdf?sequence $=1 \&$ isAllowed =y. Accessed on 08 August 2020 .

3. Mental health. Available at: https://www.who.int/health-topics/mentalhealth\#tab=tab1. Accessed on 11 August 2020.

4. World Health Organization. Mental Health. A call for action by World Health Ministers. Available at: http://www9.who.int/mental_health/media/en/249.pd f. Accessed on 12 August 2020.

5. Richardson TE, O'Reilly CL, Chen TF. Drug-related problems and the clinical role of pharmacists in inpatient mental health: an insight into practice in Australia. Int J Clin Pharm. 2014;36(5):1077-86.

6. Sarkar P, Chakraborty K, Misra A, Shukla R, Swain SP. Pattern of psychotropic prescription in a tertiary care center: A critical analysis. Ind J Pharmacol. 2013;45(3):270-3.

7. Mann E, Haastert B, Böhmdorfer B, Frühwald T, Iglseder B, Roller-Wirnsberger R et al. Prevalence and associations of potentially inappropriate prescriptions in Austrian nursing home residents: secondary analysis of a cross-sectional study. Wien Klin Wochenschr. 2013;125:180-88.

8. Hussain F, Alam M, Mali B, Akter H, Arafat SMY. Pattern of psychotropic prescription in a tertiary care hospital of Bangladesh: a cross-sectional analysis of prescription photos. Ment Health Addict Res. 2018;3(2):1-3.

9. Alam MT, Maruf MM, Sarkar M, Ahmed HU, Akhter M. Pattern of prescribing psychotropics in the outpatient department of a tertiary psychiatric hospital. Bang J Psychiatry. 2015;29(1):10-3.

10. Perumal VM, Boudhh SK, Nirmal SR, Deshpande A, Singh J, Prabhu NM. Drug utilization study and prescribing patterns in psychiatry patients at a tertiary care hospital. Int J Basic Clin Pharmacol. 2018;7(4):774-7.

11. Ubedulla SSID, Niloofar V. Drug Utilization study of psychotropic drugs in psychiatry out-patient department of a tertiary care hospital in Kerala: a geriatric perspective. Int $\mathrm{J}$ Basic Clin Pharmacol. 2019;8(8):1788-92.

12. Thakkar KB, Jain MM, Billa G, Joshi A, Khobragade AA. A drug utilization study of psychotropic drugs prescribed in the psychiatry outpatient department of a tertiary care hospital. J Clin Diagn Res. 2013;7(12):2759-64.

13. Chawla S, Agarwal M, Sharma S, Jiloha RC. Drug Utilization Study of Psychotropic Drugs among
Psychiatric Outpatients in a Tertiary Care Hospital. Indian J Pharm Sci. 2017;79(6):1008-13.

14. Sushma HK, Jyothi CH, Somashekar HS, Avanthi E, Imran M, Raja B. Prescribing pattern of antipsychotic medications in patients with schizophrenia in a tertiary care hospital. Int $\mathbf{J}$ Basic Clin Pharmacol. 2015;4(1):134-8.

15. Paul PK, Konwar M, Das S. To study the prescribing pattern of antipsychotic drugs in a tertiary care hospital of Assam. Int $\mathbf{J}$ Pharm Pharm Sci. 2014;6(4):435-7.

16. Kumar S, Chawla S, Bimba HV, Rana P, Dutta S, Kumar S. Analysis of Prescribing Pattern and Techniques of Switching Over of Antipsychotics in Outpatients of a Tertiary Care Hospital in Delhi: A Prospective, Observational Study. J Basic Clin Pharma. 2017;8:178-84.

17. Siddhartha G, Swati B, Kumar DC. Antipsychotic prescribing pattern in a tertiary care hospital of Eastern India. J Drug delivery Therap. 2013;3(4):3842.

18. Mohamed MMA, Yousef BA. Prescription patterns of antipsychotics in the management of first episode psychosis at three psychiatric hospitals in Khartoum, 2018: A descriptive cross-sectional study. J Family Med Prim Care. 2020;9(1):402-6.

19. Grover S, Nebhinani N, Chakrabarti S, Avasthi A, Mattoo SK, Basu D et al. Evaluation of psychotropic prescription patterns at the time of discharge from inpatient unit of a tertiary care general hospital psychiatric unit. J Mental Health Hum Behav. 2016;21:48-54.

20. Nukala S, Komaram RB, Singisetti S. A study on prescribing pattern of antipsychotics in Schizophrenia at a tertiary care hospital. IJPSR. 2019;10(5):2628-32.

21. Jena M, Mishra S, Mishra SN, Mishra SS. Psychotropic drugs: Prescribing pattern in psychiatry outpatient department of a tertiary care teaching hospital. Int J Pharm. 2014;4(4):204-8.

22. Sabu L, Yacob M, Mamatha K, Singh H. Drug utilization pattern of psychotropic drugs in psychiatry outpatient department in a tertiary care teaching hospital. Asian J Pharm clin Res. 2017;10(1):259-61.

23. Mukherjee S, Sen S, Chatterjee S, Biswas A, Sinha S, Ghosal et al. Prescribing Pattern of Psychotropic Medications in Psychiatry Outpatients at a Tertiary Care Teaching Hospital in India: A Prospective Cross-sectional Study. Int J Hospital Res. 2014;3(3):113-22.

24. Rode SB, Ajagallay RK, Salankar HV, Sinha U. A study on drug prescribing pattern in psychiatry putpatient department from a tertiary care etaching hospital. Int J Basic Clin Pharmacol. 2014;3(3):51722.

25. Mogali SM, Kotinatot BC. Drug utilization study of antipsychotics among schizophrenia patients in a tertiary care teaching hospital: a retrospective 
observational study. Int J Basic Clin Pharmacol. 2020;9(6):971-4.

26. Siddiqui RA, Shende TR, Mahajan HM, Borkar A. Antipsychotic medication prescribing trends in a tertiary care hospital. Int $\mathrm{J}$ Basic Clin Pharmacol. 2017;5(4):1417-20.

27. Chuki P, Khanapure A, De Sousa A. A study on prescribing patterns of atypical antipsychotics in psychiatric disorders. Int J Pharma Res Health Sci. 2014;2(4):302-6.
28. Ramadas S, Kuttichira P, Sumesh TP, Ummer SA. A study of an antipsychotic prescription pattern of patients with schizophrenia in a developing country. Indian J Psychol Med. 2010;32(1):13-6.

Cite this article as: Shareef J, Rao PGM, Ayman IM. Trends and practices in prescribing antipsychotropic medications in hospitalized patients with psychiatric disorders in a secondary care hospital. Int J Basic Clin Pharmacol 2020;9:1784-91. 